\title{
Cytoprotection Alternatives for Cancer Treatment: In-vitro Evaluation of Alpinia Zerumbet as a Radioprotective Agent
}

\author{
Bhardwaj Shukla Ritwiz ${ }^{{ }^{*}}$, Dr. Ganesh N ${ }^{2}$, Dr. Kori M.L. ${ }^{3}$ \\ 1,2 Jawaharlal Nehru Cancer Hospital and Research Center, Bhopal, India \\ ${ }^{3}$ Department of Pharmacy RKDF University, Bhopal India
}

\begin{abstract}
Cancer radiotherapy and modern synthetic medicine are critical for the treatment of cancer patients, but on the other hand they may have tremendous harmful side effect from the point of view of a weakened immune system. The focus of current research efforts in the industry and academia is not only to find affordable treatment methodologies but also to discover sources that mitigates or prevents the negative impact of these treatments on the patient's immune system. This review will provide details of optimal methodology involving Invitro application of techniques such as phytochemical analysis, thin layer chromatography, cytogenetic analysis that were adopted to assess the efficacy of Alpinia Zerumbet extracts as a radioprotector. These methodologies provide a holistic evaluation technique for assessing any potential cytoprotective agent, especially if it's from the herbal domain. As a case study, analytical and inferential results summary of the research conducted to assess radioprotective property of Alpinia Zerumbet, a herbal and readily available derivative, will be presented. The key findings from the research indicate that the Alpinia Zerumbet extract has potential to be an effective radioprotector, with minimal side effects as compared to synthetic chemical cytoprotective agents.
\end{abstract}

Keywords: Radioprotection; cancer; herbal; Alpinia Zerumbet

Article Info: Received 13 June 2019; $\quad$ Review Completed 19 July 2019; $\quad$ Accepted 24 July 2019; Available online 15 August 2019

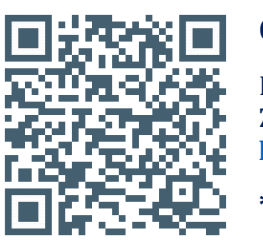

\section{Cite this article as:}

Bhardwaj Shukla R, Ganesh N, Kori ML, Cytoprotection Alternatives for Cancer Treatment: In-vitro Evaluation of Alpinia Zerumbet as a Radioprotective Agent, Journal of Drug Delivery and Therapeutics. 2019; 9(4-s):171-176 http://dx.doi.org/10.22270/jddt.v9i4-s.3268

Bhardwaj Shukla, Ritwiz, Jawaharlal Nehru Cancer Hospital and Research Center, Bhopal, India

\section{INTRODUCTION}

Cancer is known medically as a malignant neoplasm, is a broad group of various diseases, all involving unregulated cell growth. In cancer, cells divide and grow uncontrollably, forming malignant tumors, and invade nearby parts of the body. Global estimates for 2018, which cover 36 different cancers in 185 countries, report 18.1 million new cancer cases and approximately 9.6 million cancer-related deaths.[1] Rates are rising as more people live to an old age and as mass lifestyle changes occur in the developing world. There are many types of cancer treatment such as surgery, transplantation, gene, harmonal, chemo and radio therapy. Radiation therapy is one of the most common treatments for cancer. It is a local treatment which affects cancer cells only in the treated area. It is the medical use of ionizing radiation, generally as part of cancer treatment to control or kill cancerous cells using high-energy particles or waves, such as $\mathrm{x}$-rays, gamma rays, electron beams, or protons, to destroy or damage cancer cells. It is also called radiotherapy, irradiation, or x-ray therapy. Radioprotective agents are commonly used to minimize the harmful side-effects of radiotherapy treatment. Most of the currently used radioprotective agents are derived synthetically and significantly increase the cost of cancer treatment. The driver for the presented research is to discover the potential of Alpinia Zerumbet, a herbal source, which is affordable, safe and effective in minimizing the side of effects of commonly used cancer treatments of radiotherapy and also demonstrate the optimal evaluation technique that may be applied to evaluate the radioprotective properties of any chemical compound.

\subsection{Radiotherapy Mode of Action}

Radiotherapy is commonly applied to the cancerous tumor because of its ability to control cell growth. Ionizing radiation works by damaging the DNA of exposed tissue leading to cellular death. It is also common to combine radiation therapy with surgery, chemotherapy, hormone therapy, immunotherapy or some mixture of the four. Radiation involves the use of ionizing radiation to either cure or improve the symptoms of cancer. It is used in about half of all cases and the radiation can be from either internal sources in the form of brachytherapy or external sources. Radiation is typically used in addition to surgery and or chemotherapy and found effective for painful bone metastasis in about $70 \%$ of people. With the increasing technical power of radiotherapy to safely increase local tumor control for many solid tumors, it is an opportune time to rigorously explore 
the potential benefits of combining radiotherapy with molecular targeted agents and immunotherapies to increase cancer survival outcomes [2].

The basic mechanism of action of radiotherapy is by damaging the DNA of cancerous cells. This DNA damage is caused by one of two types of energy, photon or charged particle. This damage is either direct or indirect ionization of the atoms which make up the DNA chain. Indirect ionization happens as a result of the ionization of water, forming free radicals, notably hydroxyl radicals, which then damage the DNA.

Radiation therapy uses a special kind of high-energy beam to damage cancer cells. (Other types of energy beams include light and x-rays.) These high-energy beams, which are invisible to the human eye, damage a cell's DNA. Cancer cells are less organized than healthy cells, it's harder for them to repair the damage done by radiation. Therefore, cancer cells are more easily destroyed by radiation, while healthy, normal cells are better able to repair themselves and survive the treatment. Radiation can come from a machine (external radiation). It can also come from an implant (a small container of radioactive material) placed directly into or near the tumor (internal radiation). Conformal radiotherapy and Intensity modulated radiotherapy (IMRT) are example of external source. Radioactive liquids, Radioactive implants (brachytherapy) are examples of internal radiation source. Radiotherapy is an important modality and provides benefit to cancer patients, particularly in cases where surgical intervention is advisable, for example, lung, head, and neck cancers. On the other hand, high dose of radiation results in severe side effect that often leads to discontinuation of treatment cycle [3]. In the work presented, multiple level of radiation exposures were used against which the cytoprotective evaluation was done.

\subsection{Radioprotectors}

Any drug that can provide therapeutic differentiations between cancerous and normal tissue and protect the latter would be of great utility in the clinic. The development of radio protectors is important from the point of view of improving the effectiveness of cancer treatment multifold and also for strategic reasons (planned and unplanned radiation exposure.

A full range of research and development strategies is being employed currently in hunt for safe and effective radioprotections. Restructuring or reformulating older protectants have proven efficacies but unwanted toxicities. Using nutraceuticals is only moderate protection but are essentially nontoxic. Using low dose combination of potentially toxic but effective agents, induce protection through different routes and fortifies radioprotective synergy [4]. The radio protector are agents or substances that provide protection against the toxic effect of ionizing radiation and reduce the toxicity, mutagenicity and other adverse biological effects of the ionizing radiations in the living beings. The aminothiols was the first group of identified compounds as potential radio protectors. The main types of radio protector substances are the Sulfhydrylgroups compounds, amino acids, polyamines and other compounds, antioxidant and free radicals scavenger and lastly the phytochemicals compound [5].

In view of the significant side effects and associated high cost of these treatment, alternate radioprotectors which have similar efficacy and are more cost effective are needed to make cancer treatments more accessible and effective. Herbal derivatives is one such domain which may provide relevant alternatives for radioprotection. Alpinia Zerumbet was chosen for this evaluation.

\section{OBJECTIVE: ALPINIA ZERUMBET AS A HERBAL ALTERATIVE}

Bio-prospecting and natural products drug development for cancer treatment has become an important area. Most of the cancer radiotherapeutic agents are associated with toxicity towards normal cells and tissues. Optimal dosing of cancer radiotherapeutic agents is often limited because of severe non-myelosuppressive and myelo-suppressive toxicities.

It is a continuing challenge to design therapy that is safer, effective and selective. Cytoprotective agents offer opportunities to reduce treatment related toxicity of anticancer therapy without diminution of efficacy. None of the available agents satisfy criteria for an ideal cytoprotection. This has stimulated research for discovering natural resources with immunomodulatory and cytoprotective activities.

Alpinia Zerumbet also known as shell ginger, or Pink Porcelain-lily, is commonly used as a perennial decorative garden plant. The plant has globose red fruit. Native of South- East Asia. Alpinias grew from thick fleshy roots called "rhizome", similar in appearance to the "ginger root". The plant flowers on old growth and individual flowers are reminiscent of small seashells, which accounts for the common name "shell ginger" [6].

The pharmacological action of Alpinia Zerumbet Roxb. are alterative, analgesic, anthelmintic, anti-atrabilious, blood purifier, carminative, demulcent, deobastruent, diaphoretic, diuretic, emmenagogue, expectorant, febrifuge, purgative, resolvent, sedative, and anti-inflammatory. Alpinia Zerumbet has various medicinal properties as it is an antiinflammatory and analgesic. It contains rhizomes which are useful in rheumatism and catarrhal afflictions. In afflictions of gastrointestinal tract, the drugs can be used like other volatile oils. The rhizomes exhibit antiulcer activity. The rhizomes contain dihydro 5, 6-dehydrokawain which has been reported to inhibit the aggregation of ATP release from rabbit platelets induced arachidonic acid and collagen [6]. 
Table1: Botanical Classification of Alpinia Zerumbet

\begin{tabular}{|l|l|}
\hline Kingdom & Planteae -Plants \\
\hline Sub-kingdom & Tracheobionta - Vascular plants \\
\hline Super-division & Spermatophyta - Seed plants \\
\hline Division & Magnoliophyta \\
\hline Class & Lilliopsida - Monocotelydons \\
\hline Sub-class & Zingiberadeaee \\
\hline Order & Zingiberales \\
\hline Family & Zingiberaceae-Ginger family \\
\hline Genus & Alpinia Roxb - Alpinia (P) \\
\hline Species & $\begin{array}{l}\text { Alpinia Zerumbet (Pers) B.L.Burtt \& } \\
\text { R.M.Sm. -shell plant (P) }\end{array}$ \\
\hline
\end{tabular}

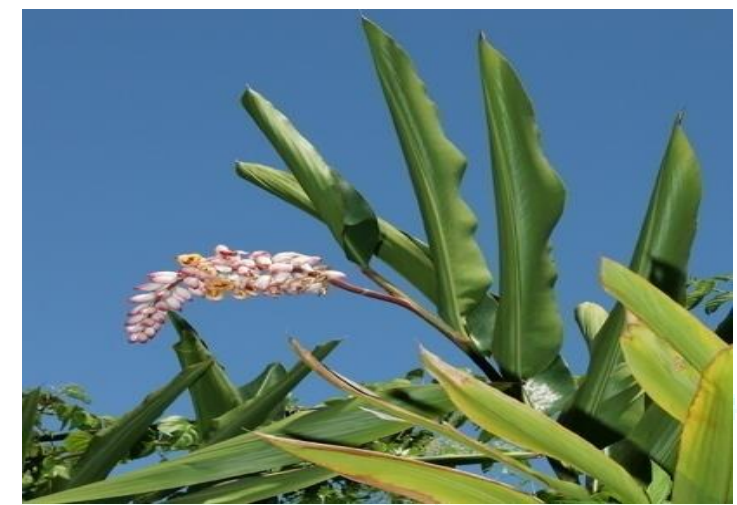

Figure 3 : Botanical Classification of Alpinia Zerumbet

Alpinia Zerumbet is a perennial plant widely distributed in subtropical and tropical regions. Consider to native north eastern India, Burma (myammar), indo-china and china and Japan. Besides it's cultivated through Southeast Asia and in many other parts[7]. The species has also been naturalized in Brazil and is also found in some areas of central and South Florida.

In terms of chemical constituents, Kava pyrone (DDK) is a medicinal compound has been detected in leaves, rhizomes, flowers and seeds of Alpinia Zerumbet [8]. It possesses several biological properties such as plant growth inhibition [18]., insecticidal activity and antifungal activity. In addition it has antiplatelet, antiulcergenic and antithrombotic effects. Some phenolic compounds have also been identified in Alpinia Zerumbet's leaves [9]. In view of the above described properties the objective of the presented work is to evaluate the radiprotective properties of Alpinia Zerumbet as a herbal radioprotector.

\section{METHODOLOGY ADOPTED}

After processing of the plant leaves, extract was prepared using maceration/ percolation technique. The solubility of the extract was evaluated with multiple solvents such as DDW, 50\% methanol, multiple DMSO concentrations: 10\%, $20 \%, 30 \%, 40 \%$ and $50 \% .50 \%$ methanol was found to be the most suitable solvent.

Next, Phytochemical analysis of the extract was done wherein, tests were done to evaluate the presence of reducing sugars, proteins, sternoid and triterpenoid, cardiac glycosides, Anthraquinone Glycosides, Cynogeneic Glycosides, Coumarin Glycoside, falvonoids, alkaloids, gums, tannins and phenolic compounds. Further Thin layer chromatography analysis was done to determine the chemical constituents of the extract.
Cytogenetic analysis was undertaken to finally evaluate the radioprotective properties of the extract against various levels of radiation (UV) exposure. Cytogenetic analyses are essential to the diagnosis and treatment of different forms of cancer, especially leukemia, cancers of the blood cell-forming system. The results of cytogenetic tests can help to confirm the diagnosis of a form of leukemia, and all the chromosomal abnormalities [10].

The cytogenetic analysis was done using cell culture. Below were the key aspects which were considered during the analysis.

1. Pre-treating cells in a hypotonic solution, which swells them and spreads the chromosomes

2. Arresting mitosis in metaphase by a solution of colchicine

3. Squashing the preparation on the slide forcing the chromosomes into a single plane

4. Cutting up a photomicrograph and arranging the result into an indisputable karyogram.

Peripheral Blood Lymphocyte culture was done with heparinized blood and using RPMI-1640 media in aseptic condition. The test samples were exposed to different duration of radiation exposure along with 50\% methanolic extract of Alpinia Zerumbet at different dosage were introduced. Drug control evaluation was set where the cultures were exposed only to various concentration of $50 \%$ methanolic extract of Alpinia Zerumbet, and only to radiation exposure for 0.5 and $1 \mathrm{hr}$. The cultured vials were incubated for 72 hours.

The cultures were then harvested for micronucleus and apoptosis assay.

The above described methodology may be utilized for any cytoprotective evaluation process.

\section{RESULTS AND DISCUSSION}

Prior to performing in vitro cytoprotective assay, phytochemicals analysis of the extract was performed and presence of different phytochemicals such as sugars, protein, steroid tri-terpiniod, cynogenetic glycosides, phenolic compound, anthraquinone, tannins flavonoids, and alkaloids was detected.

radioprotective potentiality of the extract was studied on the basis of number of micronucleus containing cells (MN cells) and apoptotic cells (AP cells) found in the peripheral blood lymphocytes culture.

To evaluate cytoprotective effect of the Alpinia Zerumbet, 5 major groups for radiooprotection analysis were taken as part of the experiment these included

To evaluate cytoprotective effect of the Alpinia zerumbet, 4 major groups for radioprotection analysis were taken as part of the experiment these included

1. NC- Normal control group (only Blood).

2. RC- Radiotherapy control group treated with UV radiation with different exposure times.

3. DC- Drug (Alpinia zerumbet extract) control group treated with 5 different concentrations of extract of Alpinia zerumbet to assess the genotoxicity of the drug.

4. R\&D- Drug and radiation therapy control group treated with different UV exposure times and drug administered either at the time of exposure or $24 \mathrm{hrs}$ after radiation exposure. 
The number of micronucleus (MN) and apoptotic (AP) cells were observed per 100 cells and scored per group. Summary of the results for each group is presented below.

1. In the case of normal control (NC) group, in which no treatment was given to the culture, no MN cells were found. $1 \%$ of the cells were seen to undergo apoptosis.

2. The radiation group $\mathrm{R} 1 \mathrm{C}$, in which cells were exposed to UV radiation for half an hour, showed $12 \%$ of MN cells and $14 \%$ apoptotic cells. In the radiation group $\mathrm{R} 2 \mathrm{C}$, in which cells were exposed to UV radiation for one hr, we found $18 \%$ of MN cells and $20 \%$ of AP cells.

3. In case of DC group, to see the genotoxic effect of extract, the culture was treated with various drug conc. i.e. $20 \%, 40 \%, 60 \%, 80 \%, 100 \%$, minimal damage to cells was observed as 3-6\% of the cells were found to contain MN cells, $60 \%$ drug conc. had maximum toxicity with $6 \%$ of MN cells, apoptosis was observed in $9 \%$, in case of $60 \%$ and $80 \%$ drug conc., $11 \%$ in $20 \%$ and $100 \%$ drug conc. and $10 \%$ in $40 \%$ drug conc.

4. In case of R\&D group, MN cells and AP cells were scored in the case of groups that were irradiated at the time of culture, with simultaneous treatment with various conc. of the drug. The groups exposed to half an hr UV radiation showed decrease in MN cells with respect to increase in drug conc. MN cells decreased to $8 \%, 6 \%, 6 \%$, and $5 \%$ in culture treated with $20 \%, 40 \%$, $60 \%$, and $80 \%$ respectively. At $100 \%$ drug conc., no MN cells were found. However, not much decrease in number of AP cells was observed 14\%, 12\%, and 15\%
AP cells were scored 20\%, 40\%, and $80 \%$ drug conc. respectively. It decreased up to $5 \%$ and $7 \%$ AP cells was seen in $60 \%$ and $100 \%$ drug conc. group.

5. The cultures exposed to UV radiation for one hr, after drug treatment were observed for $\%$ of $\mathrm{MN}$ and $\mathrm{AP}$ cells. The drug concentrations were found to exhibit considerable protective effect as the $\% \mathrm{MN}$ cells scored for conc. $20 \%, 40 \%, 60 \%, 80 \%$ and $100 \%$, the frequency of MN cells was reduced to $8 \%, 6 \%, 8 \%, 3 \%$ and $5 \%$, respectively when compared to the $18 \%$ found in radiation control group (R2C). However, not much decrease in frequency of AP cells was observed. 13\% cells were found to have undergone apoptosis in $20 \%$ and $60 \%$ drug conc. treated groups, and $10 \%, 11 \%$, and $8 \%$ were found in $40 \%, 80 \%$, and $100 \%$ drug conc. treated groups, respectively.

MN and AP cells were also scored in the culture treated with drug at the time of culture and exposed to UV radiation after $24 \mathrm{hrs}$ for duration of half an hr and one hr. A large decrease in the number of MN cells was observed in case of drug pretreated culture irradiated after $24 \mathrm{hrs}$ with UV radiation for one hr duration. Except 20\% drug conc., which had $4 \%$ MN cells, all other groups (40\%,60\%, 80\% and 100\%) were found to possess $1-2 \%$ MN cells. However, decreased in AP cells were observed with respect to increasing drug conc. $9 \%$ AP cells were found in 20\% drug conc. treated groups, $5 \%$ AP cells in case of $40 \%$ and $60 \%$ drug conc. groups, and $4 \%$ AP cells were found in respect to $80 \%$, and $100 \%$ drug conc.

Summary of the results are presented in Table 2 and statistical analysis figure $2,3,4,5$ :
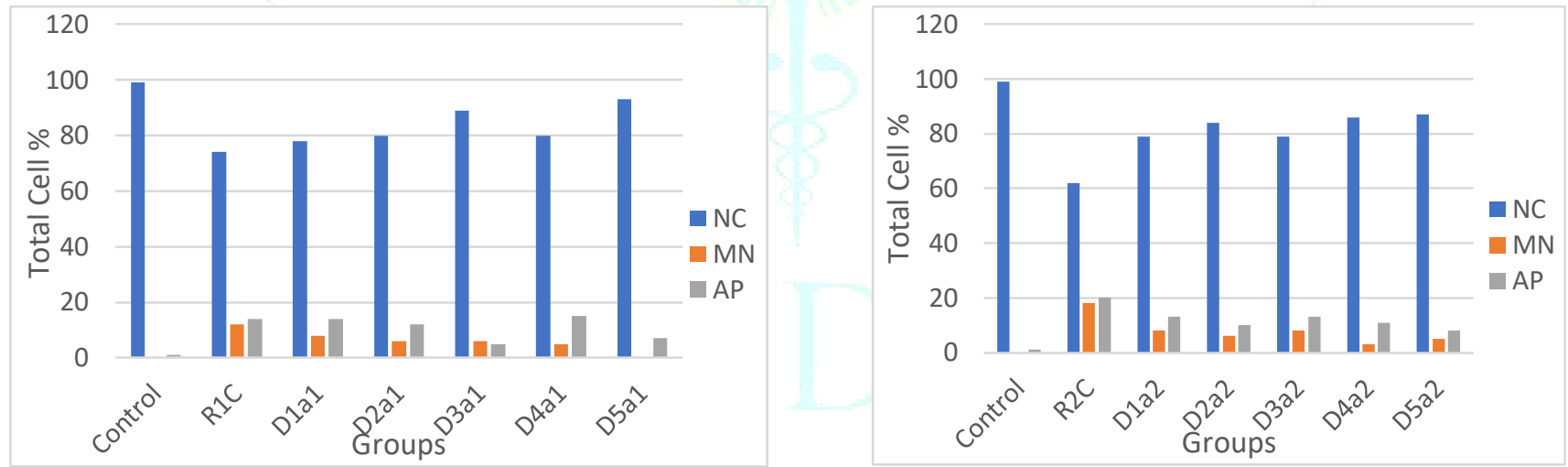

Figure 2

Figure 3

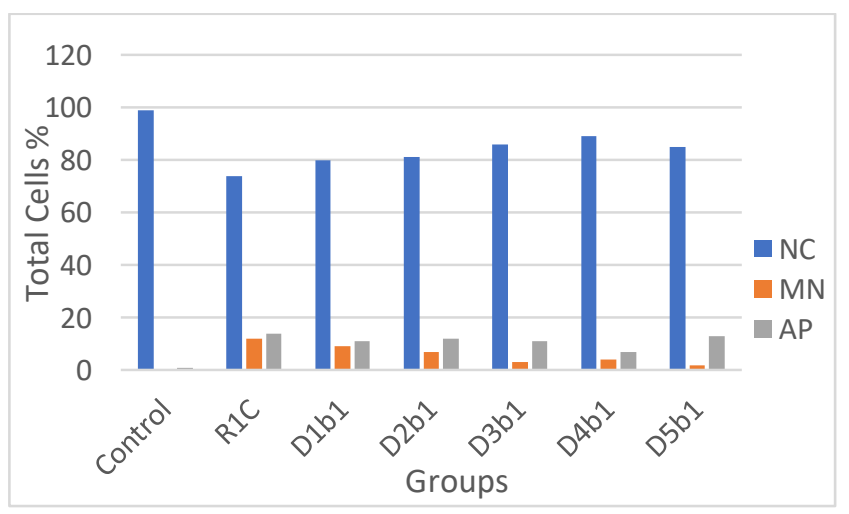

Figure 4

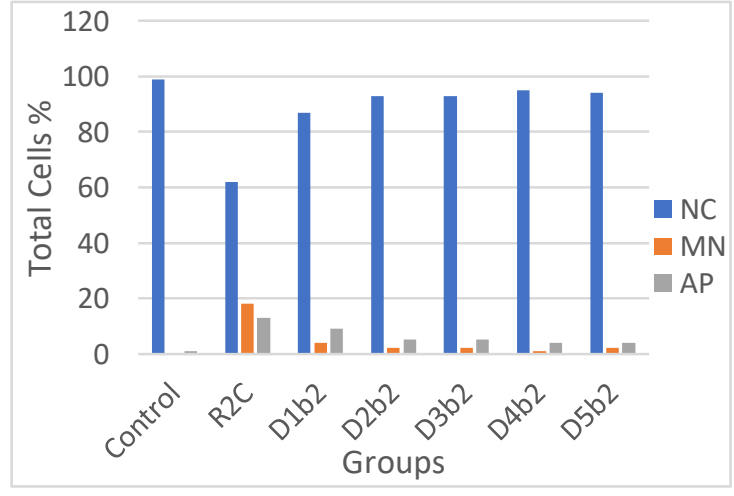

Figure 5

Figure 2: Comparison of $\mathrm{NC}, \mathrm{MN}$ and $\mathrm{AP}$ cells in control, radiation control (exposure $1 / 2 \mathrm{hr}$ ) and drug treated groups when drug is given as an adjuvant.. Figure 3: Comparison of NC, MN and AP cells in control, radiation control and drug treated group (1 hrs radiation) Figure 4 : Comparison of NC, MN and AP cells in control, radiation control (1/2 hr exposure after 24hrs of culture) and drug groups. Figure 5: Comparison of NC, MN and AP cells in radiation control and radiation drug control group (1 hrs radiation after 24 hrs of culture). 
Table 2: Represents the Micronucleus, Apoptotic cells And Total cells in In-Vitro Lymphocyte culture treated with Radiation therapy and Aqueous Extracts of Alpinia Zerumbet leaf.

\begin{tabular}{|c|c|c|c|c|c|c|}
\hline S.No. & Group & Sub Group & Total cell & $\begin{array}{c}\% \text { of Normal } \\
\text { cell }\end{array}$ & $\begin{array}{c}\% \text { of Micronucleus } \\
\text { cells }\end{array}$ & $\begin{array}{c}\% \text { of Apoptic } \\
\text { cells }\end{array}$ \\
\hline 1 & $\mathrm{NC}$ & $\mathrm{NC}$ & 100 & 99 & 0 & 1 \\
\hline 2 & \multirow{2}{*}{$\mathrm{RC}$} & R1C & 100 & 74 & 12 & 14 \\
\hline 3 & & $\mathrm{R} 2 \mathrm{C}$ & 100 & 62 & 18 & 20 \\
\hline 4 & \multirow{5}{*}{ DC } & D1 & 100 & 84 & 3 & 11 \\
\hline 5 & & D2 & 100 & 86 & 4 & 10 \\
\hline 6 & & D3 & 100 & 85 & 6 & 9 \\
\hline 7 & & D4 & 100 & 87 & 5 & 9 \\
\hline 8 & & D5 & 100 & 85 & 4 & 11 \\
\hline 9 & \multirow{20}{*}{$\mathrm{RD}$} & D1a1 & 100 & 78 & 8 & 14 \\
\hline 10 & & D2a1 & 100 & 80 & 6 & 12 \\
\hline 11 & & D3a1 & 100 & 89 & 6 & 5 \\
\hline 12 & & D4a1 & 100 & 80 & 5 & 15 \\
\hline 13 & & D5a1 & 100 & 93 & 0 & 7 \\
\hline 14 & & D1a2 & 100 & 79 & 8 & 13 \\
\hline 15 & & $\mathrm{D} 2 \mathrm{a} 2$ & 100 & 84 & 6 & 10 \\
\hline 16 & & D3a2 & 100 & 79 & 8 & 13 \\
\hline 17 & & D4a2 & 100 & 86 & 3 & 11 \\
\hline 18 & & D5a2 & 100 & 87 & 5 & 8 \\
\hline 19 & & D1b1 & 100 & 80 & 9 & 11 \\
\hline 20 & & D2b1 & 100 & 81 & 7 & 12 \\
\hline 21 & & D3b1 & 100 & 86 & 3 & 11 \\
\hline 22 & & D4b1 & 100 & 89 & 4 & 7 \\
\hline 23 & & D5b1 & 100 & 85 & 2 & 13 \\
\hline 24 & & D1b2 & 100 & 87 & 4 & 9 \\
\hline 25 & & D2b2 & 100 & 93 & 2 & 5 \\
\hline 26 & & D3b2 & 100 & 93 & 2 & 5 \\
\hline 27 & & D4b2 & 100 & 95 & 1 & 4 \\
\hline 28 & & D5b2 & 100 & 94 & 2 & 4 \\
\hline
\end{tabular}

\section{Abbreviation:}

\begin{tabular}{|l|l|}
\hline NC & No Control \\
\hline RC & Radiation Control \\
\hline DC & Drug Control \\
\hline RD & Radiation and Drug control \\
\hline R1 & $0.5 \mathrm{hr}$ radiation exposure \\
\hline R2 & $1 \mathrm{Hr}$ Radiation exposure \\
\hline D1,D2,D3,D4,D5 & $10 \%, 20 \%, 30 \%, 40 \%, 50 \%$ drug conc. \\
\hline a1 & Drug treatment at time of exposure \\
\hline a2 & Drug treatment $24 \mathrm{hr}$ after exposure \\
\hline
\end{tabular}

\section{CONCLUSIONS}

Radioprotective potential of alcoholic extract of Alpinia Zerumbet against various exposure levels of radiation was conducted.

The effect of UV radiation on lymphocytes was studied. Also, radio protective effect of drug was studied to determine whether it has better protective activity as an adjuvant to radiation exposure or when it is administered prior to the exposure.

The study shows that with increase in drug concentration, that radio protective effect exerted against UV radiation increases. When the drug was administered as an adjuvant, considerable decrease in the number of micronucleus containing cells was observed, for both the radiation exposure levels of half hour and one hour. This shows that the drug was effective in preventing chromosomal damage caused by radiation. However, it was not effective enough in prevention of the radiation induced apoptosis of cells.

In the study, when the lymphocytes were pretreated with the drug $24 \mathrm{hrs}$ before the radiation exposure, the drug was found to be effective in preventing chromosomal damage as well as induction of apoptosis. However, the protective effect was observed to be more in case of long exposure time, rather than the short half an hour exposure to UV radiation.

This protective effect of the Alpinia Zerumbet leaf extract can be attributed to the presence of compound like flavonoids and phenolics, which are known to possess free radical scavenging activity. 


\section{CONFLICT OF INTEREST}

None

\section{ACKNOWLEDGEMENT}

The authors acknowledge the support extended by the department of research, Jawaharlal Nehru cancer hospital and research center \& the department of pharmacy, Ram Krishna Dharmarth Foundation University.

\section{REFERENCES}

1. Bray, F., Ferlay, J., Soerjomataram, I., Siegel, R.L., Torre, L.A., Jemal, A., Global cancer statistics 2018: GLOBOCAN estimates of incidence and mortality worldwide for 36 cancers in 185 countries, 2018

2. Prof Robert G Bristow, Brian Alexander, Michael Baumann, Combining precision radiotherapy with molecular targeting and immunomodulatory agents: a guideline by the American Society for Radiation Oncology. The Lancet Oncology Volume 19, Issue 5, May 2018

3. Brizel DM., Wasserman TH, Henke M., Strand V., Rudat V., Monniar A., Sauer R, Phase III Radiomized trail of amifostine as a radioprotector in head and neck cancer J clin oncol, 2000, 18 (19); 3339-45.

4. Karbownik M, Reiter R.J., antioxidant effect of melatonin in protection against cellular damage, caused by ionizing radiation, Proc soc Exp Bio med. 225; 2010, 9-22.
5. Agrawal P.K., Goel H.C., Protective effect of RH-3 with special reference to radiation-induced micronuclei in mice bone marrow, 40; 2002, 525-530.

6. Pullaiah T. : "Encyclopedia of Word Medicinal Plants", Regency Publications , New Delhi, Vol.I , 2006, p.124-127.

7. Elzaawely, A.A. et al., Essential oils, kava pyrones and phenolic compounds from leaves and rhizomes of Alpinia zerumbet (Pers.) and their antioxidant activity. Food Chemistry, 2007, 103 (2); 486-494.

8. Yun-pengJiab, Ting-yu Shiac,Yan-yan ,Zhanga Dan, Lina Kegang, Linghua Yi-ni, Xua Ling Tao,Qing Lud, Xiang-chun Shena "Essential oil from Fructus Alpinia zerumbet (fruit of Alpinia zerumbet (Pers.) Burtt.et Smith) protected against aortic endothelial cell injury and inflammation in vitro and in vivo": Journal of Ethnopharmacology Volume 237, 2019, Pages 149158

9. Elzaawely, A.A., T.D. Xuan and S. Tawata : "Antioxidant activity and contents of essential oil and phenolic compounds in flowers and seeds of Alpinia zerumbet (Pers.) B.L. Burtt. and R.M. Sm.”. Food Chem., 104: 2007, 1648-165

10. Rosalind J Hastings, Nick Bown, Maria G Tibiletti, Maria Debiec-Rychter, Roberta Vanni, Blanca Espinet, Nadine van Roy, Paul Roberts, Eva van den Berg-de-Ruiter, Alain Bernheim, Jacqueline Schoumans, Steve Chatters, "Guidelines for cytogenetic investigations in tumours" European Journal of Human Genetics, 2015,: 42-48 